\title{
Towards a Federation of Smart City Services
}

\author{
José Ángel Carvajal Soto, Otilia Werner-Kytölä, \\ Marco Jahn, Jaroslav Pullmann \\ User-Centered Ubiquitous Computing department \\ Fraunhofer Institute for Applied Information Technology FIT \\ Sankt Augustin, Germany \\ \{carvajal,werner,marco.jahn,jaroslav.pullmann\}@fit.fraunhofer.de
}

\author{
Dario Bonino, Claudio Pastrone, Maurizio Spirito \\ Pervasive Technologies department \\ Istituto Superiore Mario Boella \\ Turin, Italy \\ \{bonino,pastrone,spirito\}@ismb.it
}

\begin{abstract}
The emergence of the Internet of Things has accelerated the development of smart cities. Currently, an increasing number of smart city applications comprising various scenarios and use cases is being deployed and tested. However, to realize a smart city, it is necessary to have converging solutions as opposed to niche, detached solutions. Smart city platforms need to take into account existing services and infrastructures. Furthermore, in the context of a smart city it is important to account for various kinds of stakeholders with a variety of requirements with regard to data ownership, legal and organizational frameworks as well as commercial interests. We present the ALMANAC Smart City Platform as a solution which handles the described heterogeneity. Firstly, the platform allows the development of smart city applications by creating interoperability among devices and services. Secondly, the platform is deployed in a de-centralized, federated way that allows to map the organizational requirements to the deployed city systems. We introduce the concept of federation for smart cities and describe its implementation in the ALMANAC Platform through a case study which simulates a scenario in the waste management domain. This scenario models realworld requirements of a waste management company and the municipality of Turin.
\end{abstract}

Keywords-internet of things; smart city; federation;

\section{INTRODUCTION}

Cities are densely populated urban areas where industry and/or services dominate the local economy. In general, cities agglomerate a group of interests, infrastructure, services, etc. both public and private. This group is often clustered into complex structures that are loosely interconnected and rarely share a common goal. It is often owned as well as used by a variety of entities. A city is a heterogeneous structure which mainly shares urban space and public infrastructure with the stakeholders who live in it and those who provide services to it. A smart city is an extension of a city which allows the intelligent use of resources to improve the quality of life in urban areas. Various aspects are considered to be in the focus of the transformation towards smart cities, e.g. traffic congestion, energy consumption, waste management, smart parking, smart lighting, and many more [1]. The widespread adoption of affordable, connecting technologies such as wireless sensor technologies, usually summarized under the term "Internet of Things" (IoT) [2] accelerates the process of transformation.

However, building smart city infrastructures and applications involves adding and integrating new IoT devices as much as integrating existing infrastructure and services. Considering the variety of entities in a city, that may want to become part of the smart city, there is also a need to address these entities' responsibilities, obligations, interests and infrastructures. Typically, when huge amounts of data become available, questions regarding data ownership and provisioning of services arise.

As an example, a waste management company may have an agreement with a municipality about mutual data exchange and service access to improve waste collection in the city. At the same time, the municipality may have a similar agreement with the city's water management company. But the companies themselves may be completely independent, they may not have any mutual agreement. Also the three organizations may have different contractual, legal or business restrictions regarding which data can be shared and how to share it.

In this paper we present the ALMANAC Smart City Platform with emphasis on federation. ALMANAC aims at providing a platform that solves the problem of interoperability of systems in the context of a Smart City not only at technological level, but also in regards to organizations and their regulations and policies. In allowing de-centralized, federated deployments, the platform creates the conditions needed for the already existing systems to work together, while still making it possible for them to work independently.

The remainder of this papers is as follows: In section II we present related work with regard to smart city deployment and platforms. Section III introduces the concept of federation for smart city infrastructures. In section IV we give a brief overview of the ALMANAC Smart City Platform's software architecture and present a case study of a federated deployment based on a real environment in the smart city Turin in section V. We conclude the paper with an outlook on future work in section VI. 


\section{RELATED WORK}

This section presents the related work in two parts, namely one concerning deployments, the other concerning technologies and platforms. A comparison with our work is given at the end of each part.

1) Smart City Deployments: Although smart city is already a hot topic in industries and research alike, experiences from actual deployments of smart city technologies and applications are rather scarce. One of the few examples is Smart Santander [3], a platform that serves as testbed to conduct experiments and test smart city applications, covering more than 1000 sensors. Gil et al. report experiences from their deployment of the panOULU wireless network and a corresponding middleware that should enhance communication between citizens and the government [4]. Hielkema et al. focus on developing mobile applications and services utilizing open data from municipal organizations in the Helsinki region [5]. Zanella et al. describe a deployment of a smart street lighting solution in Padova Smart City, which e.g. monitors the operation of the light bulbs [1].

The collection of smart city deployments presented here is not meant to be complete. What we want to emphasize is the lack of approaches which tackle the smart city problem as a whole rather than those which try to solve parts of the problem.

2) Smart City Technologies and Platforms: The idea of smart cities is closely linked to the concepts of the "Internet of Things" (IoT), i.e. the massive adoption of interconnected physical objects, devices, etc. [2]. IoT is considered an enabler for smart cities. Research has led to some important results and guidelines on how to realize large-scale IoT applications. The most prominent one is the "IoT-A Architectural Reference Model for the Internet of Things" [6] which provides a collection of generic architectural concepts and constructs to guide the design of IoT system architectures. The ALMANAC Smart City Platform is aligned with the IoT-A reference architecture.

Zanella et al. provide a comprehensive overview of technologies of different layers in the protocol stack from sensor technologies to web services [1]. To account for the heterogeneity in IoT and smart city environments, a set of software design decisions and principles seems reoccurring in different platform approaches. On the lowest level (i.e. physical devices) this is the abstraction of resources (e.g. [7], [8]) which is also defined by IoT-A [6]. Furthermore, orchestration, virtualization and service management as well as a minimum set of services are considered important features of a smart city platform (e.g. [9], [10]). These common approaches and services are reflected in the ALMANAC Smart City Platform's architecture design, which is described in section IV and is presented with more detail in [11].

Besides the challenges of achieving interoperability among heterogeneous technologies and services, only few research groups take into account problems such as data ownership and exchange between the different smart city stakeholders. Mitton et al. reflect on these challenges, proposing a centralized vision based on "Cloud of Things" to take care of policies for data management [12]. In contrast to centralized cloud solutions, we propose a de-centralized, federated deployment of smart city infrastructure, systems and applications.

The ALMANAC Smart City platform aims to tackle both challenges, (i) the technological challenges of achieving interoperability among heterogeneous devices and services and (ii) the legal, administrative and political aspects of smart city deployments.

\section{FEDERATION FOR SMART Cities}

Cities are heterogeneous in several aspects and already possess extensive infrastructure. Service owners, managers and legal responsibility may vary between cities and even within the same city. This situation leads to requirements for ICT systems to allow different entities to collaborate, while at the same time being able to work independently, autonomously as well as resiliently [13]. Furthermore, it requires systems that are able to incorporate existing ICT infrastructures and systems. Therefore, a smart city platform should be an integration platform. Finally, a smart city platform should be adaptable to regulations and policies of the cities as they change.

In this context, the ALMANAC Smart City Platform adapts the concept of cloud federation [14], reflecting the reality of the cities. Generally speaking, cloud federation is the interoperability of different cloud deployments as they were one, without each deployment loosing the control of its own system. Federation is more than a technical solution. It allows the stakeholders to work together as well as to work independently when it is most convenient. It enables the stakeholders to reflect public policies and regulations in their smart city systems, allowing the systems to adapt to the evolution of the city. Autonomy, integration, cooperation, resilience and the ability to adapt to regulations and policies are the advantages delivered by the federation concept.

\section{The AlmANAC SMART City Platform}

This section provides an overview of the software architecture of the ALMANAC Smart City Platform. A more comprehensive description can be found in [11]. Figure 1 shows the different parts of an ALMANAC Platform Instance (PI). A single PI consists of the following components:

- The Smart City Resource Abstraction Layer (SCRAL) enables interoperability among heterogeneous devices, wireless sensor networks and external systems. Technology-specific protocol and data formats are made available to the other platform components. 


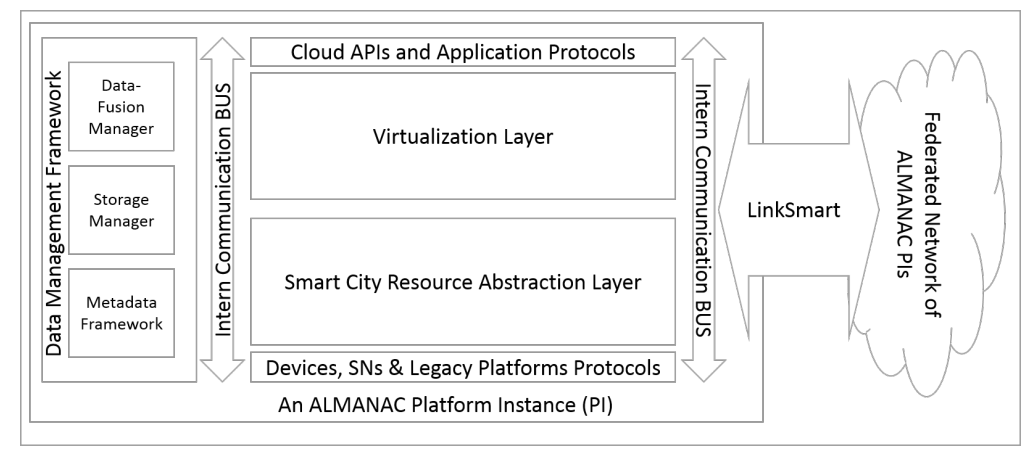

Figure 1. ALMANAC Smart City Platform Software Architecture

- The Data Management Framework provides services for stream processing (Data-Fusion Manager), data persistency (Storage Manager) and management of metadata (Metadata Framework).

- The Virtualization Layer is responsible for interfacing with high-level applications, hiding the complexity and transforming the platform protocols into applications protocols. APIs for developers are provided through ALMANAC's Cloud APIs and Application Protocols.

All components within a single Platform Instance are interconnected using either MQTT [15] or HTTP as transport mechanism and OGC SensorThings [16] as data format. MQTT is a publish-subscribe based light weight messaging protocol for use on top of the TCP/IP protocol.

Federation is managed through the distributed deployment of PIs like shown in figure 2. The intercommunication between PIs is established by the LinkSmart ${ }^{\circledR}$ Middleware [17]. It hides the complexity of the network and establishes connections in the federation. The incoming connections between PIs in federations are controlled by each PI through policies using eXtensible Access Control Markup Language (XACML) [18]. Authentication between PIs is done using SAML [19]. The access control into the platform is determined by three entry points: SCRAL, Virtualization Layer and LinkSmart ${ }^{\circledR}$ using Policy Enforcement Point (PEP) as XAML standard proposal (see section V-C for implementation details). All components of the platform mentioned above are open source, the insights and knowledge acquired by the development of the platform is shared with the community. This openness gives the ALMANAC platform added value in contrast to closed platforms.

\section{Case Studies}

The ALMANAC Smart City Platform will be deployed in several case studies in the city of Turin. These case studies are driven by application scenarios of different stakeholders, outlining the diversity of requirements for a technological smart city solution. We handle three groups of independent stakeholders with different aims and requirements regarding smart city applications and systems: the municipality, the local waste management provider, and the water service provider company. In the broader sense we also incorporate users: citizens, public officers, company employees, etc. At the same time these stakeholders are independent, they also need to share information and expose services to one another. In the following we will elaborate on this scenario as well as on how the ALMANAC platform contributes to creating a federated smart city infrastructure.

\section{A. Inter-stakeholders Waste Management Scenario}

According to Navigant Research, Smart Collection is expected to see a fast growth with annual revenue increasing at a $14.6 \%$ Compound annual growth rate (CAGR) between 2014 and 2023 [20].

ALMANAC's Waste Management solution reflects the technological state-of-the-art for the sector [20]: RFID technology for support of waste collection, waste recycling, and Pay-as-You-Throw program, as well as waste collection based on filling level and temperature sensors installed into waste bins combined with GPS systems, thereby providing the means for real-time route planning and optimization. Evidence from Europe reported in [21] suggests that waste collection costs can be reduced by up to $40 \%$ when applying solutions based on these technologies.

Concerning the waste management scenario, the interplay between the different smart city stakeholders municipality, waste management company and citizen, is in the focus of attention. A citizen passing by an overfilled waste bin would be given the possibility of reporting the issue to the city. A municipality employee would verify the issue to be able to confirm that it has not been reported yet. After the confirmation, the employee would let the waste management provider know about the new issue. The provider's waste management solution detects that the new issue is located in the vicinity of an already existing collection route assigned to a driver. The system generates an alarm to communicate the lorry driver about the new issue. The driver chooses to acknowledge the route update and to take care of the issue. After that, the citizen is informed that the issue has been handled successfully. 


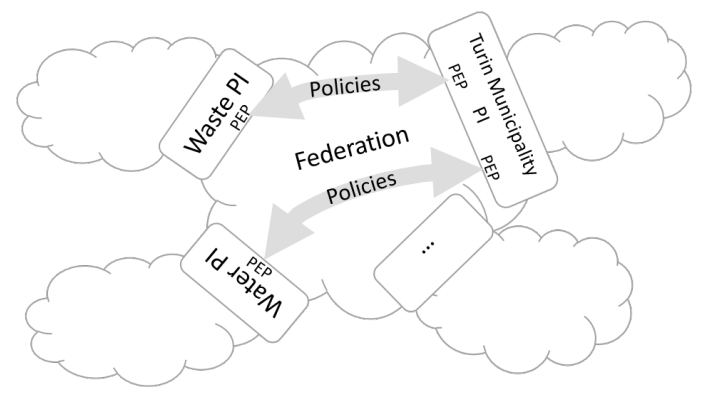

Figure 2. Federated Platform Instances

\section{B. Implementation}

The ALMANAC waste management solution prototype has its focus on waste collection, route optimization as well as on citizen engagement.

The CitizenApp as well as the DriverApp are native Android applications. The former is available for citizens to report issues to the municipality. It allows to take a picture and add a description of the situation. The latter is available for the lorry driver to follow the collection route as well as to stay up-to-date regarding real-time route optimization options. SmartWaste is a front-end application used by the municipality to manage issues reported by citizens in addition to those automatically generated by the ALMANAC Smart City platform. BeWaste is the Java backend application for issue and route management. It has an interface to the ALMANAC platform and to SmartWaste as well as to the citizen and driver apps. It is responsible for the data management aiming at guaranteeing responsiveness to the front-end applications. The applications intercommunicate through MQTT. The interface between the waste management solution and the ALMANAC platform itself is based on the Cloud APIs.

\section{Federated Deployment}

The interconnectivity of the heterogeneous ICT systems of a variety of entities and stakeholders is ensured by XACML policies controlled in each PI, like shown in figure 2. This sets the ground to each PI to itself control which operation is allowed to an external PI. The control is enforced in the requested PI by Policy Enforcement Point (PEP) inside of LinkSmart ${ }^{\circledR}$ which in turn checks if a given request is allowed. Therefore, each PI has full sovereignty over the data as well as over the operations.

A citizen who uses ALMANAC's CitizenApp to send a picture of abandoned waste will implicitly trigger the creation of an issue into the platform instance of the City of Turin. After a municipality employee has verified the issue through the SmartWaste application, it will either be marked as a duplicate of an already existing issue or it will proceed to be handled. Its handling will follow after the issue is shared through the federation with the PI of

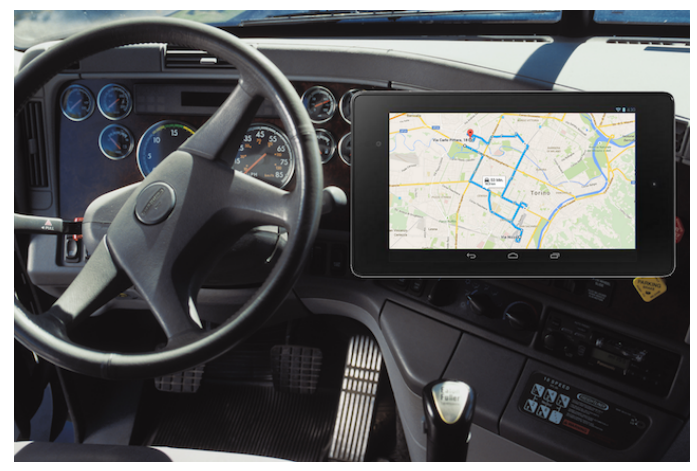

Figure 3. Scenario with the DriverApp

the waste management provider, for example when detected that the issue is located near to an already existing route which is being taken care of. In this case, the BeWaste back-end will publish a message which will be read by the DriverApp (see figure 3) of the lorry driver taking care of the route. The driver can choose through voice control to add the corresponding extra collection point to the current route and have the route be re-generated. After the route has been taken care of, BeWaste will publish a message on the succesful handling of the issue. The message will be read by the CitizenApp of the citizen who originally reported the issue and has chosen to track it.

\section{Preliminary Results}

The current deployment of the waste management comprises two Platform Instances, one simulating the waste management company and one at the municipality.

As of today, our deployment consists of real waste bins as well as of simulated bins. Real data is coming from underground waste containers, which are available to occupants of blocks of buildings. First tests are performed with eight filllevel sensors and two card readers deployed at underground waste containers affecting around 400 families. To validate the ALMANAC platform from the technical perspective, a large amount of simulated waste bins is included in the deployment. Our waste company Platform Instance includes around 28,000 waste bins for the city of Turin. In addition to these, the ALMANAC Platform's capability of dealing with large amounts of data from heterogeneous devices is validated through additional 2,000 sensors from Santander (ES) and Brussels (BE), and about 250 smart bins from Dublin (IR).

Now that the technical validation is considered successful and we have a stable deployment, we are elaborating with the municipality and the waste company on how to realize a real-world case study. The first step would be the distribution of the CitizenApp to a limited number of Turin citizens. The issue management application at the municipality would be tested to gather knowledge on the usage of the CitizenApp. 
In parallel we are exploiting possibilities and issues arising from the organizational and legal agreements that need to be implemented between the entities of such scenario. The early results of these exercises already reveal issues and requirements that are highly relevant for smart city deployments. For example, from a city's perspective regulations regarding operation in public spaces and related privacy issues need to be considered. Furthermore, existing processes such as waste collection in a large city are not changed easily. On the other hand, new business models can be developed, e.g. for dynamic taxation. In such model, citizens will pay waste taxes based on their real waste production as opposed to payment based on average costs, as in the current model. This in turn creates incentives to reducing the total waste production. We will further investigate these possibilities and issues by iterative tests and deployments as described above.

\section{CONCLUSION}

In this paper we presented the ALMANAC Smart City Platform and a federated deployment comprising two entities in the city of Turin, namely the waste management company and the municipality. First results emphasize the need for de-centralized, federated deployments to be able to account for the requirements arising due to organizational, legal, and political circumstances. Successful smart city platforms must be able to address these requirements and technologically reflect the constant evolution of smart cities, while at the same time offering an open solution which the community can leverage.

As next steps we will extend the deployment of the described use case, involving citizens via the CitizenApp. For the future, we aim at deploying the complete interstakeholders waste management scenario, not just from the ICT perspective but also considering the organizational aspects between the stakeholders.

\section{ACKNOWLEDGMENT}

The work presented in this paper was supported by the European research project ALMANAC (Project no. 609081).

\section{REFERENCES}

[1] A. Zanella, N. Bui, A. Castellani, L. Vangelista, and M. Zorzi, "Internet of things for smart cities," Internet of Things Journal, IEEE, vol. 1, no. 1, pp. 22-32, 2014.

[2] L. Atzori, A. Iera, and G. Morabito, "The internet of things: A survey," Computer networks, vol. 54, no. 15, pp. 2787-2805, 2010 .

[3] L. Sanchez, L. Muñoz, J. A. Galache, P. Sotres, J. R. Santana, V. Gutierrez, R. Ramdhany, A. Gluhak, S. Krco, E. Theodoridis et al., "Smartsantander: Iot experimentation over a smart city testbed," Computer Networks, vol. 61, pp. 217-238, 2014.
[4] F. Gil-Castineira, E. Costa-Montenegro, F. J. GonzalezCastano, C. López-Bravo, T. Ojala, and R. Bose, "Experiences inside the ubiquitous oulu smart city," Computer, no. 6, pp. 48-55, 2011.

[5] H. Hielkema and P. Hongisto, "Developing the helsinki smart city: the role of competitions for open data applications," Journal of the Knowledge Economy, vol. 4, no. 2, pp. 190204, 2013.

[6] S. Lange, A. Nettstraeter, S. Haller, F. Carrez, and A. Bassi, Eds., Introduction to the Architectural Reference Model for the Internet of Things. IoT-A Project, 2013.

[7] F. Pramudianto, I. R. Indra, and M. Jarke, "Model driven development for internet of things application prototyping," Proceedings of the 25th International Conference on Software Engineering and Knowledge Engineering, pp. 703-708, 2013.

[8] S. Alam, M. M. Chowdhury, and J. Noll, "Senaas: An event-driven sensor virtualization approach for internet of things cloud," in Networked Embedded Systems for Enterprise Applications (NESEA), 2010 IEEE International Conference on. IEEE, 2010, pp. 1-6.

[9] J. Lee, S. Baik, and C. Lee, "Building an integrated service management platform for ubiquitous cities," Computer, no. 6 , pp. 56-63, 2011.

[10] A. Krylovskiy, M. Jahn, and E. Patti, "Designing a smart city internet of things platform with microservice architecture," in The 3rd International Conference on Future Internet of Things and Cloud (FiCloud2015). IEEE, 2015.

[11] D. Bonino, M. T. Delgado Alizo, A. Alapetite, T. Gilbert, M. Axling, H. Udsen, J. A. Carvajal Soto, and M. Spirito, "Almanac: Internet of things for smart cities," in The 3rd International Conference on Future Internet of Things and Cloud (FiCloud2015). IEEE, 2015.

[12] N. Mitton, S. Papavassiliou, A. Puliafito, and K. S. Trivedi, "Combining cloud and sensors in a smart city environment," EURASIP journal on Wireless Communications and Networking, vol. 2012, no. 1, pp. 1-10, 2012.

[13] European Comission, "European Innovation Partnership on Smart Cities and Communities: Strategic Implementation Plan," http://ec.europa.eu/eip/smartcities/files/sip_final_en.pdf, 2013, accessed: July, 2015.

[14] A. Celesti, F. Tusa, M. Villari, and A. Puliafito, "How to enhance cloud architectures to enable cross-federation," in $3 r d$ International Conference on Cloud Computing. IEEE, 2010, pp. 337-345.

[15] MQTT, "MQ Telemetry Transport," http://mqtt.org, 2015, accessed: July, 2015.

[16] Open Geospatial Consortium, "OGC SensorThings API," https://ogc-iot.github.io/ogc-iot-api/, 2015, accessed: July, 2015.

[17] LinkSmart, "LinkSmart Middleware," https://linksmart.eu/, 2015, accessed: July, 2015. 
[18] XACML, "OASIS eXtensible Access Control Markup Language (XACML) TC," https://www.oasisopen.org/committees/xacml/, 2015, accessed: July, 2015.

[19] SAML, "OASIS Security Services (SAML) TC," https://www.oasis-open.org/committees/security/, 2015, accessed: July, 2015.
[20] N. Research, "Smart waste advanced collection, processing, energy recovery, and disposal technologies for the municipal solid waste," Navigant Research, Tech. Rep., 2014.

[21] H. R. Schindler, N. Schmalbein, V. Steltenkamp, J. Cave, B. Wens, and A. Anhalt, "Smart trash: Examining the impact of rfid tags in the recycling industry," RAND Corporation, Tech. Rep., 2012. 